This document is the accepted manuscript version of the following article: Hatnean, M. C., Sibille, R., Lees, M. R., Kenzelmann, M., Ban, V., Pomjakushin, V., \& Balakrishnan, G. (2017). Single crystal growth, structure and magnetic properties of Pr2Hf207 pyrochlore. Journa1 of Physics: Condensed Matter, 29(7), 075902 (9 pp.). https://doi.org/10.1088/1361-648X/29/7/075902

\title{
Single crystal growth, structure and magnetic properties of $\mathrm{Pr}_{2} \mathrm{Hf}_{2} \mathrm{O}_{7}$ pyrochlore
}

\author{
Monica Ciomaga Hatnean \\ Department of Physics, University of Warwick, Coventry, CV4 7AL, UK \\ E-mail: M.Ciomaga-Hatnean@warwick.ac.uk
}

\section{Romain Sibille}

Laboratory for Scientific Developments and Novel Materials, Paul Scherrer Institut, 5232 Villigen PSI, Switzerland

E-mail: romain.sibille@psi.ch

\section{Martin R. Lees}

Department of Physics, University of Warwick, Coventry, CV4 7AL, UK

\section{Michel Kenzelmann}

Laboratory for Scientific Developments and Novel Materials, Paul Scherrer Institut, 5232 Villigen PSI, Switzerland

\section{Voraksmy Ban}

Laboratory for Synchrotron Radiation - Condensed Matter, Paul Scherrer Institut, 5232 Villigen PSI, Switzerland

\section{Vladimir Pomjakushin}

Laboratory for Neutron Scattering and Imaging, Paul Scherrer Institut, 5232 Villigen PSI, Switzerland

\section{Geetha Balakrishnan}

Department of Physics, University of Warwick, Coventry, CV4 7AL, UK

20 September 2016

\begin{abstract}
Large single crystals of the pyrochlore $\mathrm{Pr}_{2} \mathrm{Hf}_{2} \mathrm{O}_{7}$ have been successfully grown by the floating zone technique using an optical furnace equipped with high power Xenon arc lamps. Structural investigations have been carried out by both synchrotron X-ray and neutron powder diffraction to establish the crystallographic structure of the materials produced. The magnetic properties of the single crystals have been determined for magnetic fields applied along different crystallographic axes. The results reveal that $\mathrm{Pr}_{2} \mathrm{Hf}_{2} \mathrm{O}_{7}$ is an interesting material for further investigations
\end{abstract}


as a frustrated magnet. The high quality of the crystals produced make them ideal for detailed investigations, especially those using neutron scattering techniques.

Submitted to: J. Phys.: Condens. Matter

\section{Introduction}

Pyrochlore oxides have been a topic of great interest due to the intriguing magnetic properties that originate from their frustrated magnetic lattice $[1,2,3,4,5,6,7]$. Compounds of the general formula $A_{2} B_{2} \mathrm{O}_{7}$ (where $A$ and $B$ are metals), the pyrochlores, have a face-centered cubic structure with space group $F d \overline{3} m$ (No. 227). The majority of the pyrochlore oxides belong to the $(3+, 4+)$ type $A_{2}^{3+} B_{2}^{4+} \mathrm{O}_{7}$, where the $A$ sites are occupied by trivalent cations located in the centre of scalenohedra (distorted cubes) of oxygen anions, and the $B$ sites are occupied by tetravalent transition metal ions situated in the centre of oxygen octahedra [8]. $A$ and $B$ cations form, independently, two socalled pyrochlore lattices of corner-sharing tetrahedra [3, 4]. Depending on the nature and strength of the magnetic moment and interactions, the pyrochlore oxides can display a wide variety of magnetic ground states [4] ranging from spin ice $[9,2,10]$ where the spin correlations lead to a Coulomb phase [11, 12] with emergent magnetostatics $[13,14]$, spin frozen states, [15, 16, 17] to long-range ordered states (see [4] and references therein).

Additionally, one of the most intriguing areas of current research is into materials which exhibit Quantum Spin Liquid (QSL) ground states, as has been pointed out in recent studies of pyrochlores based on $\mathrm{Yb}^{3+}[18,19,20]$, although this case is under debate $[21,22], \operatorname{Pr}^{3+}[23,24,25,26,27]$ or $\mathrm{Ce}^{3+}[28]$. The candidate materials for the realization of QSL states are based on the rare-earth ions that develop relatively small magnetic moments. The reason for this is that small magnetic moments can give the opportunity for the energy of the quantum exchange interactions (usually weak between local $4 f$ magnetic moments) to dominate over the energy of the classical dipolar interactions (proportional to the magnetic moment squared).

The synthesis of large, high quality single crystals of pyrochlore oxides, and in particular the rare-earth titanates [29, 30, 31], some rare-earth molybdates, [32, 33] and zirconates $[24,34,35,36]$ has been accomplished by the floating-zone technique. This success has allowed real and rapid progress to be made in the investigation of frustrated magnets and to unearth some very interesting magnetic properties. All the members of the titanate pyrochlore family have been thoroughly investigated over the past years, whilst the molybdate $[32,33,37,38]$ and the zirconate $[24,27,39,34,40]$ series have only recently come to the attention of the research community. Recent studies report on the availability of large crystals of the frustrated pyrochlore magnet $\mathrm{Nd}_{2} \mathrm{Hf}_{2} \mathrm{O}_{7}$ [41] . The floating zone technique is ideal to produce crystals of other members of the rare-earth hafnate pyrochlores. This is particularly appealing since the structural and magnetic characteristics of the hafnate family have not yet been investigated in great detail. 
Recent studies of the intriguing magnetic properties of the praseodymium based pyrochlores, $\mathrm{Pr}_{2} \mathrm{Zr}_{2} \mathrm{O}_{7}$ [27] and $\mathrm{Pr}_{2} \mathrm{Ir}_{2} \mathrm{O}_{7}$ [42] have motivated us to embark upon the study of the analogous compound in the hafnate pyrochlore series, $\operatorname{Pr}_{2} \mathrm{Hf}_{2} \mathrm{O}_{7}$. We have succeeded in preparing, for the first time, single crystals of the praseodymium hafnate pyrochlore by the floating-zone technique. The growth of large high-quality single crystals of this oxide represents an important step in the field, and opens up a route to further investigations of this novel class of pyrochlores, with the potential to lead to an in-depth understanding of the effects of frustration in praseodymium containing pyrochlores [25]. In this paper, we report the synthesis, the structural characterization and preliminary studies of the magnetic properties of single crystals of the praseodymium hafnate pyrochlore, $\mathrm{Pr}_{2} \mathrm{Hf}_{2} \mathrm{O}_{7}$.

\section{Experimental section}

Polycrystalline samples of $\mathrm{Pr}_{2} \mathrm{Hf}_{2} \mathrm{O}_{7}$ were prepared by conventional solid state reaction. Stoichiometric quantities of the starting materials, $\mathrm{Pr}_{6} \mathrm{O}_{11}$ (Chempur, 99.999\%) and $\mathrm{HfO}_{2}$ (Chempur, 99.95\%), were mixed, ground and heated to $1300{ }^{\circ} \mathrm{C}$ for 10 hours and $1550{ }^{\circ} \mathrm{C}$ for 10 hours with intermediate grinding. The synthesized powder was thoroughly reground and then isostatically pressed into cylindrical rods, $6-8 \mathrm{~mm}$ in diameter and about 60-70 $\mathrm{mm}$ long. The resulting rods were sintered for several days in air at $1450{ }^{\circ} \mathrm{C}$ in preparation for crystal growth experiments.

Single crystals of $\mathrm{Pr}_{2} \mathrm{Hf}_{2} \mathrm{O}_{7}$ were grown by the floating-zone technique using a four-mirror xenon arc lamp optical image furnace (CSI FZ-T-12000-X_VI-VP, Crystal Systems, Inc., Japan). The growths were performed in high purity argon at a pressure of $\sim 2$ bars, using a growth rate of $18 \mathrm{~mm} / \mathrm{h}$. The feed and the seed rods were counterrotated at around $\sim 20-30 \mathrm{rpm}$. Initially, a crystal boule of $\mathrm{Pr}_{2} \mathrm{Zr}_{2} \mathrm{O}_{7}$ was used as seed and once good quality crystals of $\mathrm{Pr}_{2} \mathrm{Hf}_{2} \mathrm{O}_{7}$ were obtained, the subsequent growths were carried out using crystal boules of $\mathrm{Pr}_{2} \mathrm{Hf}_{2} \mathrm{O}_{7}$ as seeds.

Powder X-ray diffraction experiments were carried out at the Swiss Light Source (SLS) using the MS beamline (powder station) [43]. A diffraction pattern of the $\mathrm{Pr}_{2} \mathrm{Hf}_{2} \mathrm{O}_{7}$ polycrystalline material (starting material for the growth) was measured in a quartz capillary $(\phi=0.1 \mathrm{~mm})$ with the Debye-Scherrer geometry and a multistrip MYTHEN II detector. The incident beam had an energy of $\sim 22 \mathrm{keV}(\lambda=0.564941 \AA)$ and the diffracted beams were measured up to $60^{\circ} 2 \theta$, with a step size of $\sim 0.0036^{\circ} 2 \theta$. Powder neutron diffraction experiments were carried out on the starting polycrystalline material at the Swiss spallation neutron source SINQ using the HRPT diffractometer $(\lambda=1.155 \AA)$. Diffraction was measured between 5 and $162^{\circ} 2 \theta$, with a step size of $0.05^{\circ}$ in $2 \theta$, in a standard "Orange" helium cryostat. A joint Rietveld refinement with equal weighting factors for the synchrotron and neutron data was performed using the FULLPROF software suite [44]. The instrumental resolution functions were experimentally determined from the measurements of small linewidth standards. A total of 23 parameters were refined: two sets of parameters independently refined for the two 
diffraction patterns (scale factors, zero-shifts, lattice parameter, sample contributions to the peak shapes), and twelve parameters defining the structural model ( $x$ coordinate of the $48 f$ oxygen atom, anisotropic displacement parameters of all atoms, occupancy factors of three atomic positions).

In addition to the powder synchrotron X-ray diffraction pattern measured on the starting polycrystalline material, a measurement was also performed on a sample obtained by grinding a tiny crystal fragment from the middle of a single crystal. The drawbacks of using a powdered crystal fragment were the small amount of the resulting powder and the difficulty in grinding the crystal sample into very fine particles. The aforementioned factors hindered the realization of a full Rietveld analysis, because of insufficient powder averaging and poorly modelled lineshapes. Instead, that data was analyzed by a Le Bail decomposition that was good enough to provide a precise estimate of the lattice parameter.

A Laue X-ray imaging system with a Photonic-Science Laue camera was used to investigate the quality of the crystal boules and to orient single-crystal samples for selected experiments. A rectangular-prism shaped sample, with dimensions of $2.73 \times$ $1.81 \times 1.96 \mathrm{~mm}^{3}$, was cut from the $\mathrm{Pr}_{2} \mathrm{Hf}_{2} \mathrm{O}_{7}$ boule for magnetization measurements. The sample was cut in order that the [110] (rhombic) and [001] (tetragonal) directions were perpendicular to the faces of the rectangular prism. The demagnetizing factors were calculated using expressions derived by Aharoni [45].

Magnetization measurements were carried out using a Quantum Design Magnetic Property Measurement System MPMS-5S superconducting quantum interference device (SQUID) magnetometer along with an i-Quantum ${ }^{3} \mathrm{He}$ insert. The magnetic susceptibility, which is equal to the magnetization $M$ divided by the magnetic field $H$ in the linear field regime, was evaluated as a function of temperature in a constant applied magnetic field of $1 \mathrm{kOe}$ from 0.5 to $300 \mathrm{~K}$. Magnetization measurements were also performed as a function of magnetic field up to $70 \mathrm{kOe}$ directed along specific crystallographic axes at various temperatures.

\section{Results and Discussion}

\subsection{Crystal chemistry}

Firstly, we have investigated in detail the crystal structure of $\mathrm{Pr}_{2} \mathrm{Hf}_{2} \mathrm{O}_{7}$ in order to confirm the relevance of this material as a model pyrochlore magnet. We have used the polycrystalline sample prepared as starting material for the crystal growth for these experiments. Diffraction patterns were measured using synchrotron X-ray (Fig. 1a) and neutron (Fig. 1b) radiation and refined together against the pyrochlore structure (space group $F d \overline{3} m$, origin choice 2). The Rietveld procedure converges rapidly and the conventional agreement factors for Rietveld refinements [46] are $R_{\mathrm{WP}}=2.04$ and $R_{\mathrm{Bragg}}=2.66$, and $R_{\mathrm{WP}}=5.19$ and $R_{\mathrm{Bragg}}=4.24$, respectively for the synchrotron $\mathrm{X}$-ray and neutron patterns at $300 \mathrm{~K}$. 

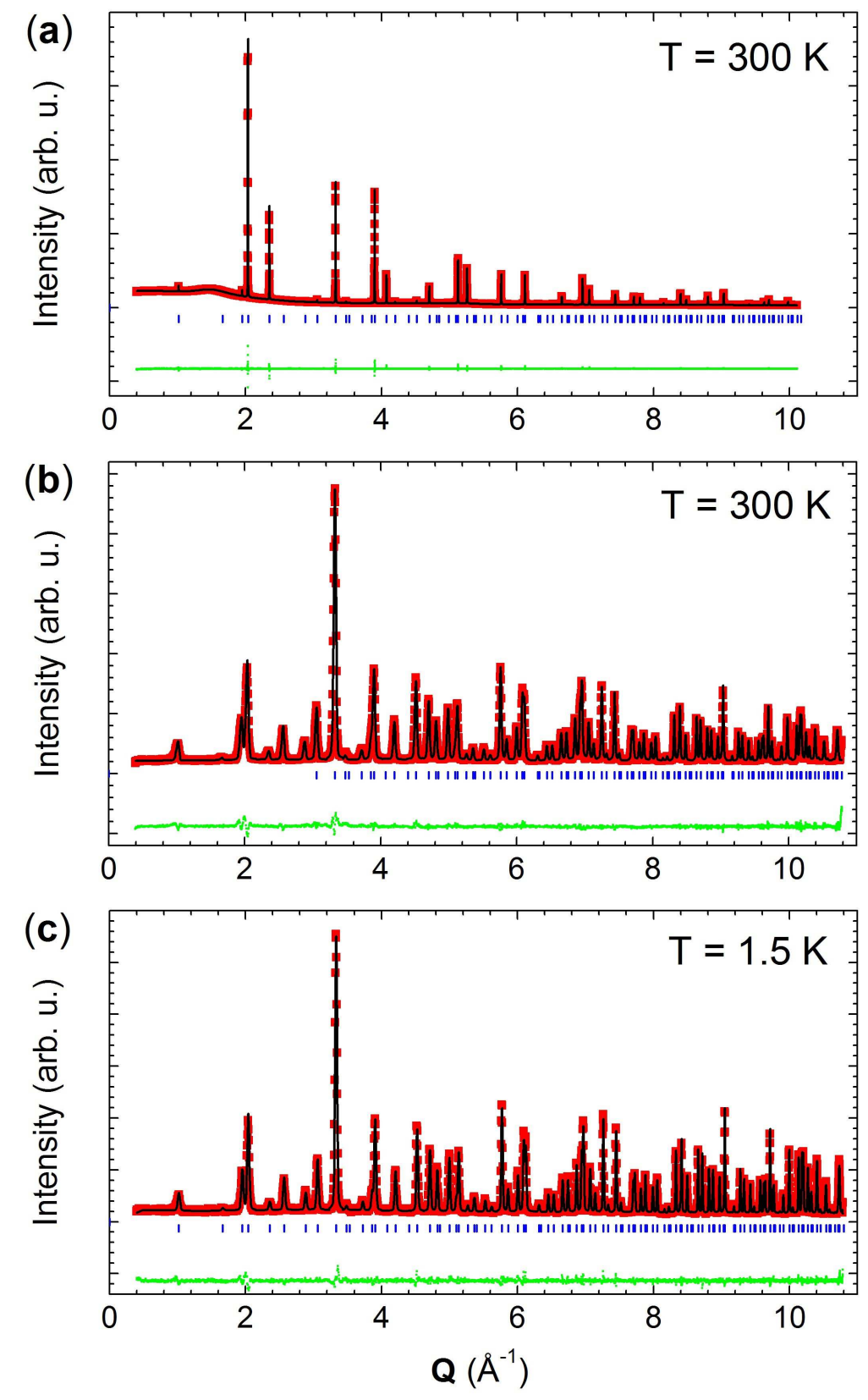

Figure 1. Rietveld refinement of powder (a) synchrotron X-ray and (b and c) neutron diffraction data collected at $300 \mathrm{~K}$ (a and b) and at $1.5 \mathrm{~K}$ (c). Black, red, and green represent the experimental data, the fit, and difference between the data and the fit respectively, while the blue ticks indicate the Bragg positions. The incident wavelengths are $\lambda=0.621418 \AA$ (a) and $\lambda=1.155 \AA$ (b and c). Patterns (a) and (b) are jointly refined against a common structural model given in Table 1. The low-temperature structure corresponding to pattern (c) is presented in Table 2. Conventional Rietveld factors for pattern (a) (\%): $R_{\mathrm{P}}=1.70 ; R_{\mathrm{WP}}=2.04 ; R_{\mathrm{Bragg}}=$ 2.66; $R_{\mathrm{F}}=7.45$. Conventional Rietveld factors for pattern (b) (\%): $R_{\mathrm{P}}=3.88$; $R_{\mathrm{WP}}=5.19 ; R_{\mathrm{Bragg}}=4.24 ; R_{\mathrm{F}}=2.58$. Conventional Rietveld factors for pattern (c) $(\%): R_{\mathrm{P}}=3.88 ; R_{\mathrm{WP}}=5.19 ; R_{\mathrm{Bragg}}=3.91 ; R_{\mathrm{F}}=2.55$. 
The joint refinement provides a complete description of the room-temperature crystal structure: precise lattice parameter, bond distances/angles and anisotropic displacement parameters (ADPs), as well as a certain degree of sensitivity to the chemical composition thanks to the strong contrast in the neutron scattering lengths $\left(b_{\mathrm{Pr}}=4.58(5) \mathrm{fm}, b_{\mathrm{Hf}}=7.77(14) \mathrm{fm}, b_{\mathrm{O}}=5.805(4) \mathrm{fm}\right)$. The results are summarized in Table 1 and are in good agreement with previously published data [47, 48]. The resulting crystal structure at $300 \mathrm{~K}$ is shown on Fig. 2. The lattice parameter obtained from the powder synchrotron X-ray data is $10.68411(2) \AA$. The value of the atomic coordinate $x$ for the oxygen atom $\mathrm{O}(48 f)$ is $0.33247(7)$, in the range of the typical values for $A_{2} B_{2} \mathrm{O}_{7}$ compounds [4]. The Pr-O(48f) bond length is 2.6016(6) $\AA$, close to the sum of the ionic radii ( $\sim 2.66 \AA$ ), while $\mathrm{Pr}-\mathrm{O}^{\prime}(8 b)$ bond (pointing along the local $\langle 111\rangle$ direction) has a length of 2.312697(6) $\AA$, which is markedly shorter than the 2.66 Ausually observed in rare-earth pyrochlores. Attempts to refine antisite cation disorder and oxygen Frenkel disorder did not provide evidence for any deviation from a perfectly ordered pyrochlore structure.
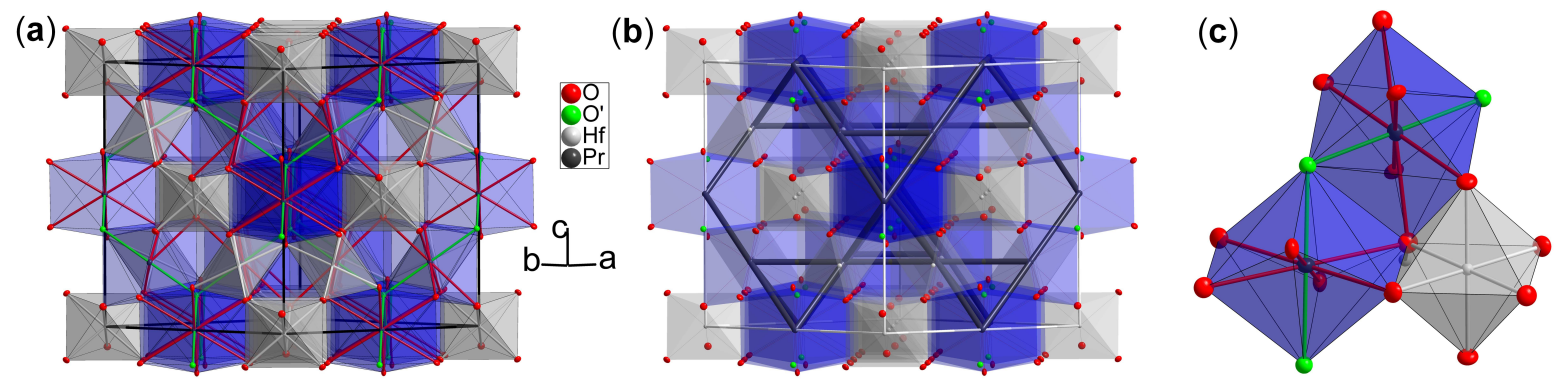

Figure 2. Crystal structure of $\mathrm{Pr}_{2} \mathrm{Hf}_{2} \mathrm{O}_{7}$ obtained from the joint Rietveld refinement of synchrotron X-ray and neutron data measured at room temperature, $F d \overline{3} m$, origin choice 2. In panel (a) the cell edges are drawn in black, and we emphasize the Pr-O (red), Pr-O' (green) and Hf-O (grey) bonds. Blue and grey polyhedra show the oxygen scalenohedra and octahedra around $\mathrm{Pr}^{3+}$ and $\mathrm{Hf}^{4+}$ cations, respectively. In panel (b) the cell edges are drawn in white and we emphasize the magnetic pyrochlore lattice of $\mathrm{Pr}^{3+}$ cations (in black). Panel (c) shows the local coordination around the two metals and the connection between the polyhedra.

The joint refinement can also be used to retrieve information concerning the composition of the sample. Three of the four occupancy factors were refined in order to avoid total correlation with the scale factor. The refined chemical occupancies remain very close to unity (see Table 1). Given this result it is reasonable to assume a stoichiometric formula $\mathrm{Pr}_{2} \mathrm{Hf}_{2} \mathrm{O}_{7}$ for our polycrystalline material.

Finally, we have also collected a powder neutron diffraction pattern of $\mathrm{Pr}_{2} \mathrm{Hf}_{2} \mathrm{O}_{7}$ at $1.5 \mathrm{~K}$, in order to determine values for the bond distances and angles that are relevant for the low-temperature superexchange pathways, and to check for structural distortions that may affect the magnetism of the non-Kramers $\operatorname{Pr}^{3+}$ ions. The crystal structure maintains its cubic symmetry at $1.5 \mathrm{~K}$, where the lattice $(a=10.66564(5) \AA)$ contracts by about $1.5 \%$ compared to its value at $300 \mathrm{~K}(a=10.68189(5) \AA$ obtained from the 
Table 1. Structural parameters for a polycrystalline sample of $\mathrm{Pr}_{2} \mathrm{Hf}_{2} \mathrm{O}_{7}$ determined from a joint Rietveld refinement of synchrotron X-ray and neutron diffraction data measured at $300 \mathrm{~K}$ (space group $F d \overline{3} m$, origin choice 2 ).

\begin{tabular}{l|cccc|cl}
\hline $\boldsymbol{T}=300 \mathbf{K}$ & $x$ & $y$ & $z$ & & Occupancy & \\
\hline $\operatorname{Pr}(16 d)$ & 0.5 & 0.5 & 0.5 & & $0.994(12)$ & \\
Hf $(16 c)$ & 0 & 0 & 0 & & 1 & \\
O $(48 f)$ & 0.375 & 0.375 & 0.375 & & $1.024(15)$ & \\
$\mathrm{O}^{\prime}(8 b)$ & $0.33247(7)$ & 0.125 & 0.125 & & $1.012(27)$ & \\
\hline ADPs in $\AA^{2}:$ & $U_{11}$ & $U_{22}$ & $U_{33}$ & $U_{12}$ & $U_{13}$ & $U_{23}$ \\
\hline Pr $(16 d)$ & 0.00702 & 0.00702 & 0.00702 & -0.00084 & -0.00084 & -0.00084 \\
Hf $(16 c)$ & 0.00355 & 0.00355 & 0.00355 & 0.00015 & 0.00015 & 0.00015 \\
O1 $(48 f)$ & 0.00966 & 0.00712 & 0.00712 & 0 & 0 & 0.00269 \\
O2 $(8 b)$ & 0.00665 & 0.00665 & 0.00665 & 0 & 0 & 0
\end{tabular}

Table 2. Structural parameters for a polycrystalline sample of $\mathrm{Pr}_{2} \mathrm{Hf}_{2} \mathrm{O}_{7}$ obtained from a Rietveld refinement of powder neutron diffraction data measured at $1.5 \mathrm{~K}$ (space group $F d \overline{3} m$, origin choice 2). Results are given assuming all the occupancy factors are equal to unity.

\begin{tabular}{l|ccclll}
\hline $\boldsymbol{T}=\mathbf{1 . 5} \mathbf{K}$ & $x$ & $y$ & $z$ & & & \\
\hline $\mathrm{Pr}(16 d)$ & 0.5 & 0.5 & 0.5 & & & \\
Hf $(16 c)$ & 0 & 0 & 0 & & & \\
O $(48 f)$ & 0.375 & 0.375 & 0.375 & & & \\
$\mathrm{O}^{\prime}(8 b)$ & $0.33301(7)$ & 0.125 & 0.125 & & & \\
\hline ADPs in $\AA^{2}:$ & $U_{11}$ & $U_{22}$ & $U_{33}$ & $U_{12}$ & $U_{13}$ & $U_{23}$ \\
\hline $\operatorname{Pr}(16 d)$ & 0.00334 & 0.00334 & 0.00334 & 0.00062 & 0.00062 & 0.00062 \\
Hf $(16 c)$ & 0.00116 & 0.00116 & 0.00116 & -0.00015 & -0.00015 & -0.00015 \\
O1 $(48 f)$ & 0.00480 & 0.00460 & 0.00460 & 0 & 0 & 0.00008 \\
O2 $(8 b)$ & 0.00419 & 0.00419 & 0.00419 & 0 & 0 & 0
\end{tabular}

refinement of the neutron diffraction pattern). The results of the refinement at $1.5 \mathrm{~K}$ which is presented in Fig. 1c, are summarized in Table 2. At $1.5 \mathrm{~K}$ the $\operatorname{Pr}-\mathrm{O}(48 f)$ bond length is 2.5937(6) $\AA$ and the Pr-O' (8b) bond has a length of 2.309179(6) $\AA$.

\subsection{Crystal growth}

Crystals of $\mathrm{Pr}_{2} \mathrm{Hf}_{2} \mathrm{O}_{7}$ were successfully grown by the floating zone method, using similar growth conditions to those used for preparing $\mathrm{Pr}_{2} \mathrm{Zr}_{2} \mathrm{O}_{7}$ crystal boules [34, 49]. One of the difficulties associated with the growth of praseodymium related compounds is the evaporation of $\mathrm{Pr}_{2} \mathrm{O}_{3}$ during the crystal growth process, which can cause a decrease in the Pr content in the single crystals [24, 34, 49]. This phenomena can be avoided by employing a high growth rate and by performing the growth in a pressurized gas atmosphere (inside a quartz tube) to suppress the evaporation [34]. The crystal growth 
of $\mathrm{Pr}_{2} \mathrm{Hf}_{2} \mathrm{O}_{7}$ was performed in high purity argon gas in order to facilitate the reduction of the $\operatorname{Pr}^{4+}$ ions to $\operatorname{Pr}^{3+}$ (see Refs. [34] and [35], and references therein). $\operatorname{Pr}_{2} \mathrm{Hf}_{2} \mathrm{O}_{7}$ crystals obtained were typically 5-7 $\mathrm{mm}$ in diameter and $60-85 \mathrm{~mm}$ long. The crystals developed well defined facets, within the first few millimeters of the growth and the boules obtained were free of any cracks. No deposition was observed on the quartz tube surrounding the sample during the growth process, suggesting that no evaporation occurred during any of the growths. All the praseodymium hafnate boules were transparent to light, with a bright green colour. A photograph of an as-grown crystal of $\operatorname{Pr}_{2} \mathrm{Hf}_{2} \mathrm{O}_{7}$ is shown in Fig. 3a. The crystal quality of the boules was investigated by Laue X-ray diffraction, and Laue photographs were taken along the length of the boule, on the faceted sides (see Fig. 3a). The Laue patterns were identical along the whole length of the faceted faces and, in most cases, the [110] direction is almost orthogonal to one of the facets. A Laue photograph taken on an aligned sample of $\mathrm{Pr}_{2} \mathrm{Hf}_{2} \mathrm{O}_{7}$ used for magnetic properties measurements is shown in Fig. $3 b$.
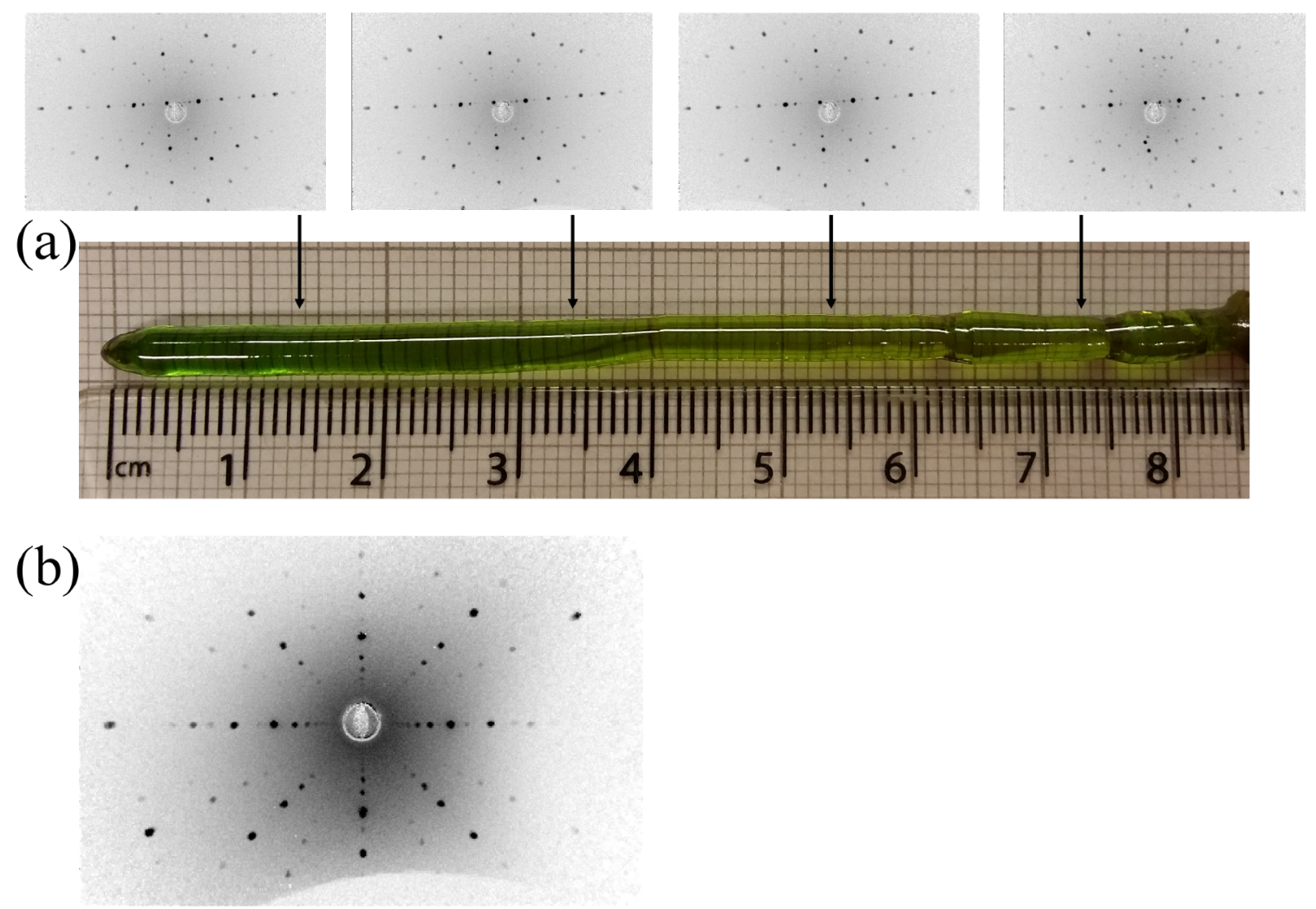

Figure 3. (a) Crystal of $\mathrm{Pr}_{2} \mathrm{Hf}_{2} \mathrm{O}_{7}$ grown in high purity argon atmosphere, at a pressure of $\sim 2$ bars and a translation rate of $18 \mathrm{~mm} / \mathrm{h}$. Also shown above the image of the crystal are the Laue patterns of one of the facets, taken along the crystal length at $\sim 2 \mathrm{~cm}$ intervals, between the end (left) and the beginning (right) of the boule. The corresponding Laue patterns taken on the facet at 180 degrees are mirror images of these patterns. (b) Laue back reflection X-ray photograph of an aligned sample (showing the [001] orientation) used for the magnetic properties measurements discussed in the present work. 
The powder synchrotron X-ray diffraction pattern of a ground fragment taken from the middle of the specimen shown in Fig. 3a was refined against the pyrochlore lattice (Fig. 4). The pattern matches very well with the cubic pyrochlore phase and no impurity peaks were present. Furthermore, the superlattice reflections which are the characteristic trademarks of the pyrochlore structure are clearly visible in the X-ray diffraction pattern. The lattice parameter (10.67704(3) $\AA$ ) was found to be slightly smaller than the value of 10.68411(2) $\AA$ obtained at the same temperature and using the same method for our polycrystalline material (Fig. 1a). A difference in the value of the lattice parameter between the polycrystalline and single crystal samples has also been reported for the $\mathrm{Pr}_{2} \mathrm{Zr}_{2} \mathrm{O}_{7}$ pyrochlore [34]. The smaller lattice parameter observed in the single crystals may be attributed to a very small difference in the stoichiometry of polycrystalline and single crystalline samples.

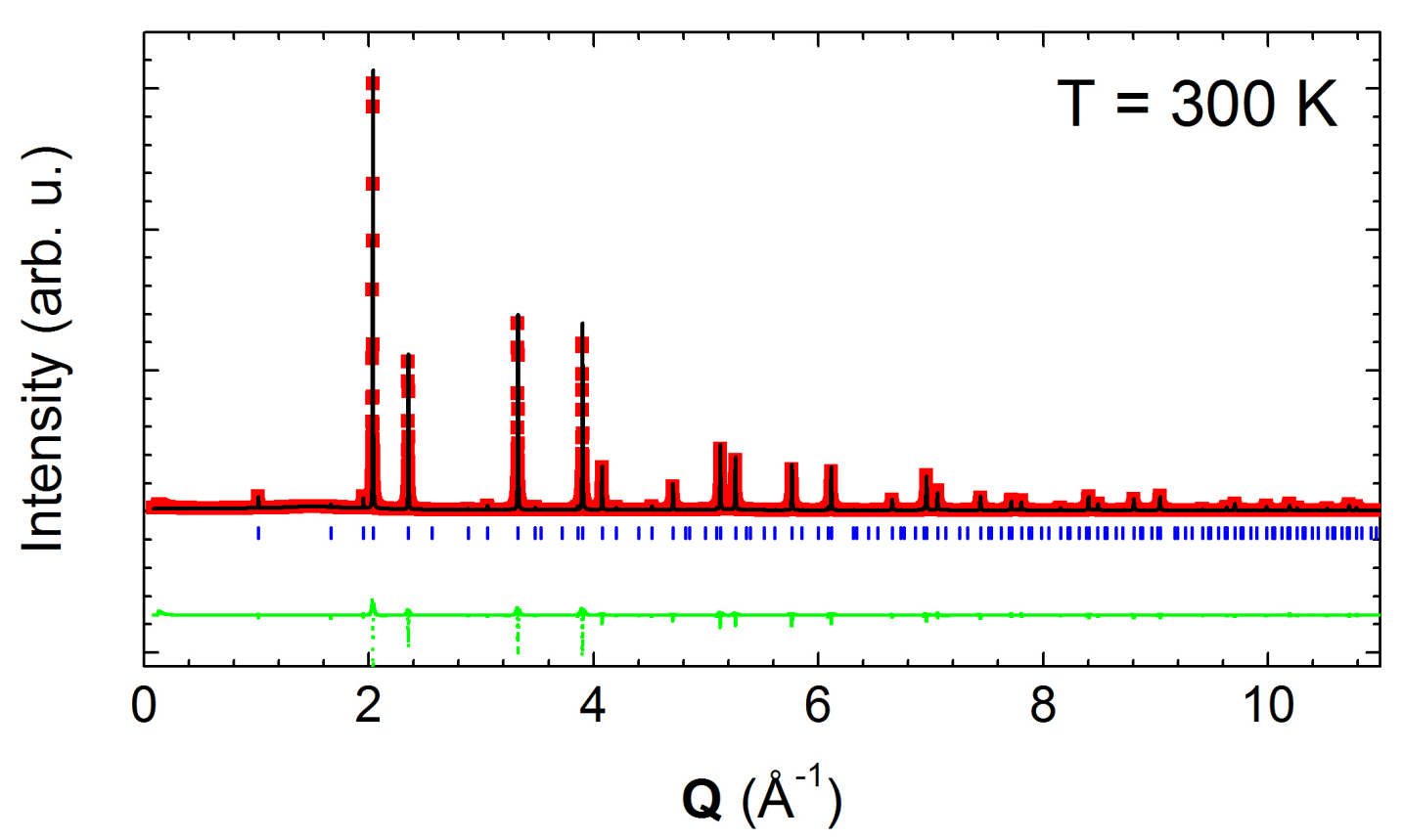

Figure 4. Powder synchrotron X-ray diffraction pattern collected on a ground crystal of $\mathrm{Pr}_{2} \mathrm{Hf}_{2} \mathrm{O}_{7}$ at $300 \mathrm{~K}$. The experimental profile (red) and a Le Bail decomposition (black) are shown, with the difference given in green. The Bragg positions are indicated by the blue ticks.

\subsection{Magnetic properties}

Field-cooled (FC) and zero-field-cooled (ZFC) magnetization versus temperature data were collected on a $\mathrm{Pr}_{2} \mathrm{Hf}_{2} \mathrm{O}_{7}$ rectangular-prism-shaped single crystal aligned along the three high symmetry crystallographic directions ([100], [110] and [111]). The data were corrected for demagnetization effects [45]; the demagnetizing factors were found 
to be equal to $N=0.38,0.35$ and 0.25 , respectively, where $H=H_{\text {applied }}-4 \pi M N$. Fig. 5 shows the temperature dependency of the $d c$ magnetic susceptibility, $\chi(T)$, and the reciprocal $d c$ magnetic susceptibility $\chi^{-1}(T)$. The data measured along the different crystallographic axes in $1 \mathrm{kOe}$ reveal a monotonic and highly-isotropic (within experimental error) increase upon cooling from $T=300$ to $0.5 \mathrm{~K}$, and the absence of any anomaly which may indicate a magnetic transition. $\chi^{-1}(T)$ data do not obey a Curie-Weiss law in the temperature range 0.5 to $300 \mathrm{~K}$, although fits could be made over a reduced temperature range $(0.5$ to $10 \mathrm{~K})$ [see Fig. 5 (b)(inset)]. It was found that the results of the fits depend on the exact temperature range over which the fit is performed. These results highlight the importance of investigating the crystal electric field (CEF) scheme in these systems. The crystal field splitting of $\operatorname{Pr}^{3+}$ in $\operatorname{Pr}_{2} \mathrm{Hf}_{2} \mathrm{O}_{7}$ was determined and the results are described elsewhere [50]. $\mathrm{In}_{\mathrm{Pr}_{2}} \mathrm{Hf}_{2} \mathrm{O}_{7}$ the first excited level is about $9.2 \mathrm{meV} \sim 107 \mathrm{~K}$ above the ground state doublet, meaning that information concerning the magnetic interactions can be deduced from a Curie-Weiss analysis well below this temperature. Accordingly, a fit of the magnetic susceptibility to a Curie-Weiss law was made in the temperature range from 0.5 to $10 \mathrm{~K}$, yielding a Curie-Weiss temperature of $\theta_{\mathrm{W}}=-0.43(1) \mathrm{K}$ for the magnetic field applied along the [111] direction. This indicates the presence of antiferromagnetic interactions, slightly weaker than in $\operatorname{Pr}_{2} \mathrm{Zr}_{2} \mathrm{O}_{7}$ where $\theta_{\mathrm{W}}=-1.4(1) \mathrm{K}[27]$. The $\operatorname{Pr}^{3+}$ effective moment is estimated to be $\mu_{\mathrm{eff}}=2.51(1) \mu_{\mathrm{B}}$, a similar value to the one found in $\mathrm{Pr}_{2} \mathrm{Zr}_{2} \mathrm{O}_{7}$ [27, 34]. The calculated values of the Curie-Weiss temperature $\theta_{\mathrm{W}}$, and of the effective moment $\mu_{\text {eff }}$ are in agreement with those determined by detailed investigations of the low temperature magnetic properties of our $\mathrm{Pr}_{2} \mathrm{Hf}_{2} \mathrm{O}_{7}$ crystals [50].
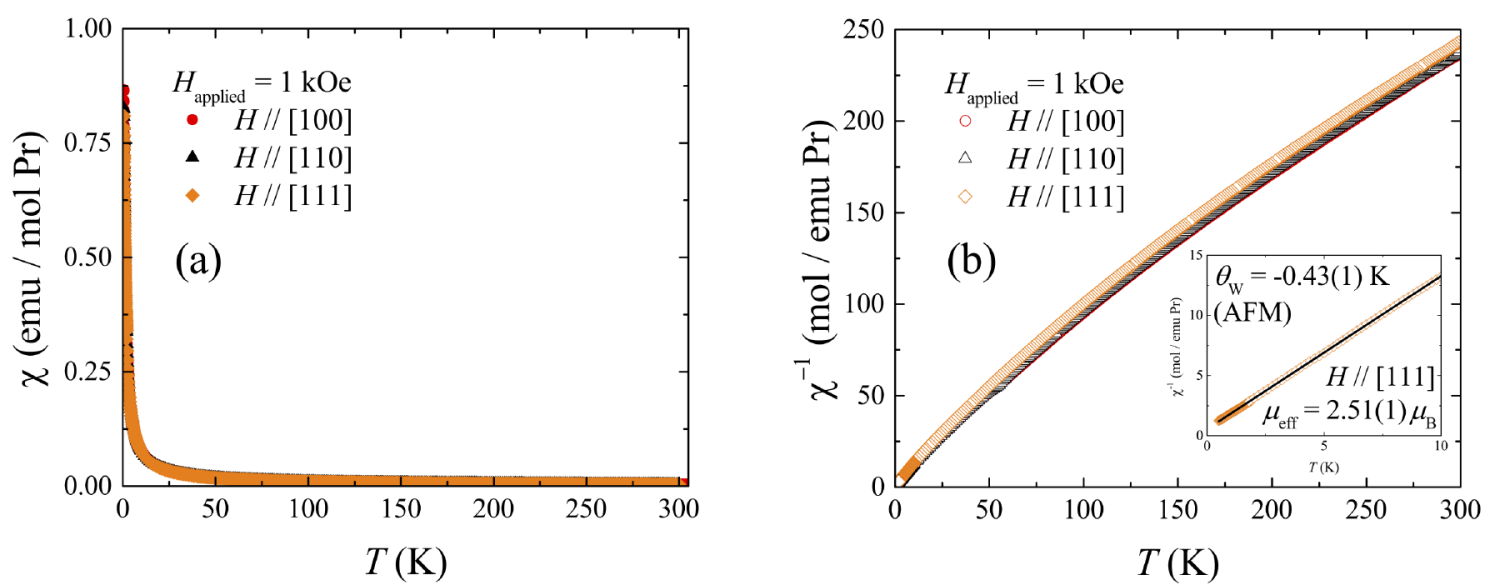

Figure 5. (a) Temperature dependence of the $d c$ magnetic susceptibility, $\chi$ versus $T$, in the temperature range 0.5 to $300 \mathrm{~K}$ for a crystal of $\mathrm{Pr}_{2} \mathrm{Hf}_{2} \mathrm{O}_{7}$, with a magnetic field applied along the [100] (red), [110] (black), and [111] (orange) directions. (b) Temperature dependence of the reciprocal of the bulk $d c$ susceptibility, $\chi^{-1}$ versus $T$, for a field applied along the three high symmetry directions. The inset shows $\chi^{-1}$ versus $T$ and the linear fit (using the Curie-Weiss law) to the data in the temperature range 0.5 to $10 \mathrm{~K}$ for a magnetic field applied along the [111] direction. 
Single crystal growth, structure and magnetic properties of $\mathrm{Pr}_{2} \mathrm{Hf}_{2} \mathrm{O}_{7}$ pyrochlore

The magnetization measured along the three directions as a function of applied magnetic field $M(H)$ at various temperatures is shown in Fig. 6. The data collected at $1.8 \mathrm{~K}$ or below [see Figs. 6(a) and 6(b)] reveal a non linear response of the magnetization as the applied field increases. Furthermore, the field dependence of the magnetization appears reversible, with no hysteresis between the field increasing and field decreasing $M(H)$ curves. The magnetization measured with a magnetic field applied along the [100] direction is the highest in strong magnetic fields, whilst the [110] direction gives the lowest values of the magnetization. These results suggest similarities in terms of local anisotropy of the magnetic moments between the $\mathrm{Pr}_{2} \mathrm{Hf}_{2} \mathrm{O}_{7}$ pyrochlore and the classical spin ice systems such as $\mathrm{Dy}_{2} \mathrm{Ti}_{2} \mathrm{O}_{7}$ and $\mathrm{Ho}_{2} \mathrm{Ti}_{2} \mathrm{O}_{7}$ [51, 52]. However, the values of the magnetic moments measured for the three crystallographic directions at the maximum applied field are smaller than the values of the expected saturated moments for a classic spin ice configuration [51] and there is no evidence of a plateau developing in the $M(H)$ curve when the field is applied along the [111] direction. A similar local $\langle 111\rangle$ Ising behaviour was also observed in the related praseodymium zirconate pyrochlore, $\mathrm{Pr}_{2} \mathrm{Zr}_{2} \mathrm{O}_{7}[27,39,34]$. In $\mathrm{Pr}_{2} \mathrm{Hf}_{2} \mathrm{O}_{7}$ the magnetization response is temperature dependent (see Figs. 6(a)-(f)), with the strongly anisotropic response only observed below about $10 \mathrm{~K}$.
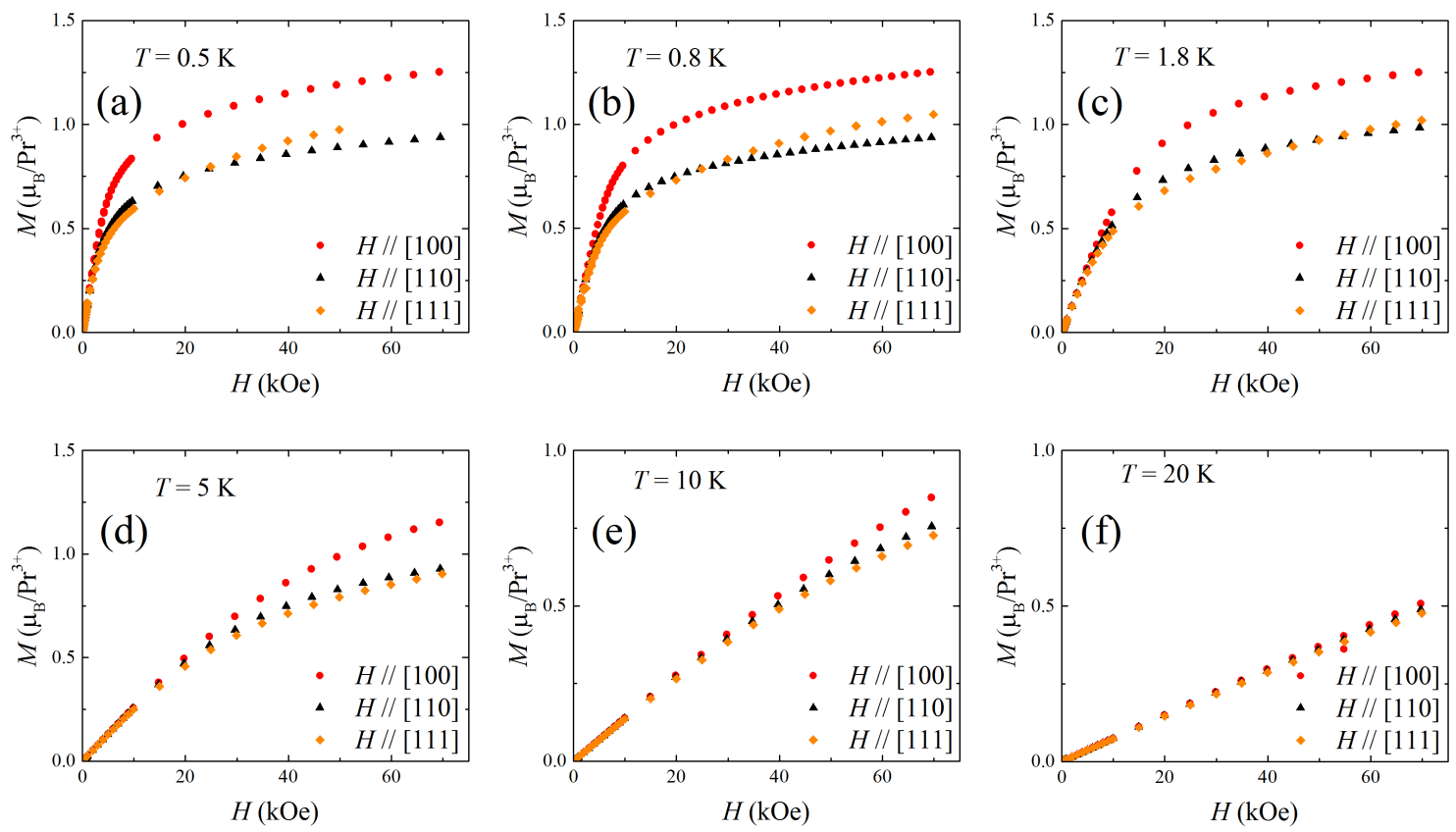

Figure 6. Isothermal magnetization $(M)$ as a function of applied magnetic field $(H)$ along the [100] (red), [110] (black), and [111] (orange) directions at temperatures of (a) 0.5 , (b) 0.8 , (c) 1.8, (d) 5, (e) 10 and (f) $20 \mathrm{~K}$ for a single-crystal of $\operatorname{Pr}_{2} \mathrm{Hf}_{2} \mathrm{O}_{7}$. 


\section{Summary}

We have successfully prepared large, high-quality single crystals of the novel frustrated pyrochlore magnet $\mathrm{Pr}_{2} \mathrm{Hf}_{2} \mathrm{O}_{7}$ by the floating zone technique, using a growth rate of $18 \mathrm{~mm} / \mathrm{h}$ in a high purity argon atmosphere, at a pressure of $\sim 2$ bars. Powder X-ray diffraction studies confirm that the crystal boules are of single-phase pyrochlore $F d \overline{3} m$ structure. The quality of our $\mathrm{Pr}_{2} \mathrm{Hf}_{2} \mathrm{O}_{7}$ single crystals appears to be very high according to several criteria (colour homogeneity, absence of cracks, transparency, quality and spatial homogeneity of the X-ray Laue diffraction patterns). The temperature dependence of the magnetic susceptibility measured in a low magnetic field shows an isotropic behaviour without any sign of long-range magnetic ordering down to $0.5 \mathrm{~K}$. The field dependence of the isothermal magnetization reveals an anisotropic behaviour at low temperature, indicating a spin ice type of anisotropy. The absence of a plateau in the field dependent magnetization when the field is applied along the [111] direction, as opposed to the classic spin ice pyrochlores $\mathrm{Dy}_{2} \mathrm{Ti}_{2} \mathrm{O}_{7}$ and $\mathrm{Ho}_{2} \mathrm{Ti}_{2} \mathrm{O}_{7}$, may be related to the quantum nature of the magnetic ground state in $\mathrm{Pr}_{2} \mathrm{Hf}_{2} \mathrm{O}_{7}$. This magnetic response is similar to that seen in the related pyrochlore praseodymium zirconate, $\mathrm{Pr}_{2} \mathrm{Zr}_{2} \mathrm{O}_{7}$. However, in contrast to the $\mathrm{Pr}_{2} \mathrm{Zr}_{2} \mathrm{O}_{7}$ pyrochlore in which recent results point to the existence of a certain degree of disorder [53], the investigations performed on our $\mathrm{Pr}_{2} \mathrm{Hf}_{2} \mathrm{O}_{7}$ crystals show a structure with no cationic or anionic deficiencies. A recent determination of the crystal field scheme in $\mathrm{Pr}_{2} \mathrm{Hf}_{2} \mathrm{O}_{7}$ using neutron spectroscopy on polycrystalline samples [50] has confirmed the nature of the anisotropy deduced for the bulk measurements presented here. The $\mathrm{Pr}_{2} \mathrm{Hf}_{2} \mathrm{O}_{7}$ crystals produced are ideal for further investigations of the low temperature magnetism by neutron scattering techniques.

\section{Acknowledgments}

This work was supported by a grant from the EPSRC, UK (Grant No. EP/M028771/1). The authors thank Mr. Tom E. Orton for valuable technical support. We acknowledge funding from the European Community's Seventh Framework Program (Grant No. 290605, COFUND: PSI-FELLOW), and the Swiss National Science Foundation (Grant Nos. 200021_138018 and 200020_162626). Neutron scattering experiments were carried out at the continuous spallation neutron source SINQ at the Paul Scherrer Institut at Villigen PSI in Switzerland and at the Institut Laue Langevin in Grenoble, France. Synchrotron powder X-ray diffraction measurements were carried out at the Materials Science beamline X04SA of the Swiss Light Source (SLS) at the Paul Scherrer Institut at Villigen PSI in Switzerland.

\section{References}

[1] Blöte $\mathrm{H}$ W J, Wielinga R F and Huiskamp W J 1969 Physica 43 549-568 URL http://www.sciencedirect.com/science/article/pii/0031891469901876 
Single crystal growth, structure and magnetic properties of $\mathrm{Pr}_{2} \mathrm{Hf}_{2} \mathrm{O}_{7}$ pyrochlore

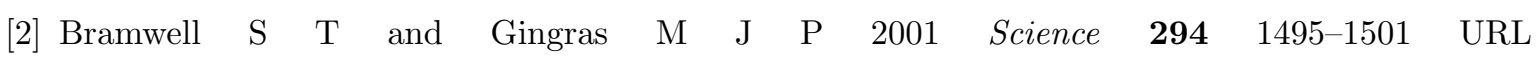
http://www. sciencemag.org/content/294/5546/1495. abstract

[3] Greedan J E 2001 Journal of Materials Chemistry 11(1) 37-53 URL http://dx.doi.org/10.1039/B003682J

[4] Gardner J S, Gingras M J P and Greedan J E 2010 Reviews of Modern Physics 82(1) 53-107 URL http://link.aps.org/doi/10.1103/RevModPhys.82.53

[5] Malkin B Z, Lummen $\mathrm{T}$ T A, van Loosdrecht $\mathrm{P}$ H M, Dhalenne $\mathrm{G}$ and Zakirov A R 2010 Journal of Physics: Condensed Matter 22276003 URL http: //stacks. iop.org/0953-8984/22/i=27/a=276003

[6] Petrenko O A, Lees M R and Balakrishnan G 2011 Journal of Physics: Condensed Matter 23 164218 URL http://stacks .iop.org/0953-8984/23/i=16/a=164218

[7] Gingras M J P and McClarty P A 2014 Reports on Progress in Physics 77056501 URL http://stacks . iop.org/0034-4885/77/i=5/a=056501

[8] Subramanian M A, Aravamudan G and Rao G V S 1983 Progress in Solid State Chemistry 15 55-143 URL http://www. sciencedirect.com/science/article/pii/0079678683900018

[9] Harris M J, Bramwell S T, McMorrow D F, Zeiske T and Godfrey K W 1997 Physical Review Letters 79(13) 2554-2557 URL http://link.aps.org/doi/10.1103/PhysRevLett.79.2554

[10] Bramwell S T, Harris M J, den Hertog B C, Gingras M J P, Gardner J S, McMorrow D F, Wildes A R, Cornelius A L, Champion J D M, Melko R G and Fennell T 2001 Physical Review Letters 87(4) 047205 URL http://link.aps.org/doi/10.1103/PhysRevLett.87.047205

[11] Henley C L 2010 Annual Review of Condensed Matter Physics 1 179-210

[12] Fennell T, Deen P P, Wildes A R, Schmalzl K, Prabhakaran D, Boothroyd A T, Aldus R J, McMorrow D F and Bramwell S T 2009 Science 326 415-417

[13] Castelnovo C, Moessner R and Sondhi S L 2008 Nature $45142-45$

[14] Castelnovo C, Moessner R and Sondhi S L 2012 Annual Review of Condensed Matter Physics 3 $35-55$

[15] Greedan J, Sato M, Yan X and Razavi F S 1986 Solid State Communications 59 895-897 ISSN 0038-1098 URL http://www.sciencedirect.com/science/article/pii/0038109886906526

[16] Gaulin B D, Reimers J N, Mason T E, Greedan J E and Tun Z 1992 Physical Review Letters 69(22) 3244-3247 URL http://link.aps.org/doi/10.1103/PhysRevLett.69.3244

[17] Zhou H D, Wiebe C R, Harter A, Dalal N S and Gardner J S 2008 Journal of Physics: Condensed Matter 20325201 URL http://stacks.iop.org/0953-8984/20/i=32/a=325201

[18] Ross K A, Savary L, Gaulin B D and Balents L 2011 Physical Review X 1021002

[19] Chang L J, Onoda S, Su Y, Kao Y J, Tsuei K D, Yasui Y, Kakurai K and Lees M R 2012 Nature Communications 3992

[20] Applegate R, Hayre N R, Singh R R P, Lin T, Day A G R and Gingras M J P 2012 Physical Review Letters 109(9) 097205 URL http://link.aps.org/doi/10.1103/PhysRevLett.109.097205

[21] Robert J, Lhotel E, Remenyi G, Sahling S, Mirebeau I, Decorse C, Canals B and Petit S 2015 Physical Review B 92(6) 064425 URL http://link.aps.org/doi/10.1103/PhysRevB.92.064425

[22] Jaubert L D C, Benton O, Rau J G, Oitmaa J, Singh R R P, Shannon N and Gingras M J P 2015 Physical Review Letters 115(26) 267208 URL http://link.aps.org/doi/10.1103/PhysRevLett.115.267208

[23] Zhou H D, Wiebe C R, Janik J A, Balicas L, Yo Y J, Qiu Y, Copley J R D and Gardner J S 2008 Physical Review Letters 101227204

[24] Matsuhira K, Sekine C, Paulsen C, Wakeshima M, Hinatsu Y, Kitazawa T, Kiuchi Y, Hiroi Z and Takagi S 2009 Journal of Physics: Conference Series 145012031 URL http: //stacks . iop.org/1742-6596/145/i=1/a=012031

[25] Onoda S and Tanaka Y 2010 Physical Review Letters 105(4) 047201 URL http://link.aps.org/doi/10.1103/PhysRevLett.105.047201

[26] Lee S, Onoda S and Balents L 2012 Physical Review B 86(10) 104412 URL http://link.aps.org/doi/10.1103/PhysRevB.86.104412 
Single crystal growth, structure and magnetic properties of $\mathrm{Pr}_{2} \mathrm{Hf}_{2} \mathrm{O}_{7}$ pyrochlore

[27] Kimura K, Nakatsuji S, Wen J J, Broholm C, Stone M B, Nishibori E and Sawa H 2013 Nature Communications 41934 URL http://dx.doi.org/10.1038/ncomms2914

[28] Sibille R, Lhotel E, Pomjakushin V, Baines C, Fennell T and Kenzelmann M 2015 Physical Review Letters 115(9) 097202 URL http://link.aps.org/doi/10.1103/PhysRevLett.115.097202

[29] Balakrishnan G, Petrenko O A, Lees M R and Paul D M 1998 Journal of Physics: Condensed Matter 10 L723 URL http://stacks.iop.org/0953-8984/10/i=44/a=002

[30] Gardner J S, Gaulin B D and Paul D M 1998 Journal of Crystal Growth 191 740-745 URL http://www.sciencedirect.com/science/article/pii/S0022024898003820

[31] Prabhakaran D and Boothroyd A T 2011 Journal of Crystal Growth 318 1053-1056 URL http://www.sciencedirect.com/science/article/pii/S0022024810010298

[32] Taguchi Y, Ohgushi K and Tokura Y 2002 Physical Review B 65(11) 115102 URL http://link.aps.org/doi/10.1103/PhysRevB.65.115102

[33] Kézsmárki I, Hanasaki N, Hashimoto D, Iguchi S, Taguchi Y, Miyasaka $\mathrm{S}$ and Tokura Y 2004 Physical Review Letters 93(26) 266401 URL http://link.aps.org/doi/10.1103/PhysRevLett.93.266401

[34] Ciomaga Hatnean M, Decorse C, Lees M R, Petrenko O A, Keeble D S and Balakrishnan G 2014 Materials Research Express 1026109 URL http: //stacks. iop.org/2053-1591/1/i=2/a=026109

[35] Koohpayeh S M, Wen J J, Trump B A, Broholm C L and McQueen T M 2014 Journal of Crystal Growth 402 291-298 URL http://www.sciencedirect.com/science/article/pii/S0022024814004217

[36] Ciomaga Hatnean M, Lees M R and Balakrishnan G 2015 Journal of Crystal Growth 418 1-6 URL http://www.sciencedirect.com/science/article/pii/S0022024815000792

[37] Hanasaki N, Kinuhara M, Kézsmárki I, Iguchi S, Miyasaka S, Takeshita N, Terakura C, Takagi H and Tokura Y 2006 Physical Review Letters 96(11) 116403 URL http://link.aps.org/doi/10.1103/PhysRevLett.96.116403

[38] Kézsmárki I, Hanasaki N, Watanabe K, Iguchi S, Taguchi Y, Miyasaka S and Tokura Y 2006 Physical Review B 73(12) 125122 URL http://link.aps.org/doi/10.1103/PhysRevB.73.125122

[39] Kimura K, Nakatsuji S and Nugroho A A 2013 Journal of the Korean Physical Society 63 719-721 URL http://dx.doi.org/10.3938/jkps.63.719

[40] Ciomaga Hatnean M, Lees M R, Petrenko O A, Keeble D S, Balakrishnan G, Gutmann M J, Klekovkina V V and Malkin B Z 2015 Physical Review B 91(17) 174416 URL http://link.aps.org/doi/10.1103/PhysRevB.91.174416

[41] Chun J, Reuvekamp P G, Chen D, Lin C and Kremer R K 2015 Journal of Materials Chemistry C 3(3) 491-494 URL http://dx.doi.org/10.1039/C4TC02416H

[42] Tokiwa Y, Ishikawa J J, Nakatsuji S and Gegenwart P 2014 Nature Materials 13 356-359 URL http://dx.doi.org/10.1038/nmat3900

[43] Willmott P R, Meister D, Leake S J, Lange M, Bergamaschi A, Bge M, Calvi M, Cancellieri C, Casati N, Cervellino A, Chen Q, David C, Flechsig U, Gozzo F, Henrich B, Jggi-Spielmann S, Jakob B, Kalichava I, Karvinen P, Krempasky J, Ldeke A, Lscher R, Maag S, Quitmann C, Reinle-Schmitt M L, Schmidt T, Schmitt B, Streun A, Vartiainen I, Vitins M, Wang X and Wullschleger R 2013 Journal of Synchrotron Radiation 20 667-682 ISSN 1600-5775 URL http://dx.doi.org/10.1107/S0909049513018475

[44] Rodríguez-Carvajal J 1993 Physica B: Condensed Matter $192 \quad 55-69$ URL http://www.sciencedirect.com/science/article/pii/092145269390108I

[45] Aharoni A 1998 Journal of Applied Physics 83 3432-3434 URL http://scitation.aip.org/content/aip/journal/jap/83/6/10.1063/1.367113

[46] McCusker L B, Von Dreele R B, Cox D E, Louër D and Scardi P 1999 Journal of Applied Crystallography 32 36-50 URL http://dx.doi.org/10.1107/S0021889898009856

[47] Karthik C, Anderson T J, Gout D and Ubic R 2012 Journal of Solid State Chemistry 194 168-172 URL http://www.sciencedirect.com/science/article/pii/S0022459612003210 
1

2

3

4

5

6

7

8

9

10

11

12

13

14

15

16

17

18

19

20

21

22

23

24

25

26

27

28

29

30

31

32

33

34

35

36

37

38

39

40

41

42

43

44

45

46

47

48

49

50

51

52

53

54

55

56

57

58

59

60

Single crystal growth, structure and magnetic properties of $\mathrm{Pr}_{2} \mathrm{Hf}_{2} \mathrm{O}_{7}$ pyrochlore

[48] Blanchard P E R, Liu S, Kennedy B J, Ling C D, Avdeev M, Aitken J B, Cowie B C C and Tadich A 2013 The Journal of Physical Chemistry C 117 2266-2273

[49] Ciomaga Hatnean M, Decorse C, Lees M R, Petrenko O A and Balakrishnan G 2016 Crystals 6 79 URL http://www.mdpi.com/2073-4352/6/7/79

[50] Sibille R, Lhotel E, Ciomaga Hatnean M, Balakrishnan G, Fåk B, Fennell T and Kenzelmann M 2016 Physical Review B 94024436 URL http://journals.aps.org/prb/abstract/10.1103/PhysRevB.94.024436

[51] Fukazawa H, Melko R G, Higashinaka R, Maeno Y and Gingras M J P 2002 Physical Review B 65(5) 054410 URL http://link.aps.org/doi/10.1103/PhysRevB.65.054410

[52] Petrenko O A, Lees M R and Balakrishnan G 2003 Physical Review B 68(1) 012406 URL http://link.aps.org/doi/10.1103/PhysRevB.68.012406

[53] Petit S, Lhotel E, Florea S G O, Robert J, Bonville P, Mirebeau I, Ollivier J, Mutka H, Ressouche E, Decorse C, Ciomaga Hatnean M and Balakrishnan G 2016 ArXiv e-prints (Preprint 1609.01391) 